\title{
The Clinical Characteristics of Acute Heart Failure Patients with Mid-Range Ejection Fraction in Turkey: A Subgroup Analysis from Journey HF-TR Study
}

\author{
Umit Yasar Sinan, Dogac Caglar Gurbuz', Oguzhan Celik², Huseyin Altug Cakmak³ Salih Kilic ${ }^{4}$, Sinan Inci5 ${ }^{5}$ Gulay Gok ${ }^{6}$, \\ Mehmet Serdar Kucukokglu, Mehdi Zoghi ${ }^{7}$
}

Department of Cardiology, Istanbul University Cerrahpasa Institute of Cardiology, Istanbul, 'Department of Cardiology, Izmir Katip Celebi University Ataturk Training and Research Hospital, Izmir, 'Department of Cardiology, Hitit University School of Medicine, Corum, ${ }^{3}$ Department of Cardiology, Mustafakemalpasa State Hospital, Bursa, ${ }^{4}$ Department of Cardiology, Gaziantep Doctor Ersin Arslan Training and Research Hospital, Gaziantep, ${ }^{5}$ Department of Cardiology, Aksaray State Hospital, Aksaray, ${ }^{6}$ Department of Cardiology, Mardin State Hospital, Mardin, ${ }^{7}$ Department of Cardiology, Ege University School of Medicine, Izmir, Turkey

ORCID:

Umit Yasar Sinan: https://orcid.org/0000-0002-4837-7099

Dogac Caglar Gurbuz: https://orcid.org/0000-0002-0517-8612

Oguzhan Celik: https://orcid.org/0000-0002-0716-8602

Huseyin Altug Cakmak: https://orcid.org/0000-0002-5101-1928

Salih Kilic: https://orcid.org/0000-0002-5138-744X

Sinan Inci: https://orcid.org/0000-0002-4576-128X

Gulay Gok: https://orcid.org/0000-0003-0205-1138

Mehmet Serdar Kucukoglu: https://orcid.org/0000-0002-3145-6209

Mehdi Zoghi: https://orcid.org/0000-0002-8156-2675

\section{Abstract}

Background: Heart failure (HF) is a clinical syndrome characterized with a wide spectrum of left ventricular (LV) structural and functional abnormalities. LV ejection fraction (EF) is considered important with respect to classifying HF patients because of differing patient demographics and prognosis; as well as the response to HF therapies. We aimed to investigate the clinical characteristics, demographics, in-hospital management and in-hospital outcome of HF patients with mid-range EF (HFmrEF) in comparison with those with HF patients with reduced EF (HFrEF) or HF patients with preserved EF (HFpEF) in a large acute HF (AHF) cohort. Materials-Methods and Results: The Journey HF-TR study is a multicenter, and observational registry. One thousand six hundred and six patients who were diagnosed with AHF were enrolled in this study. The mean age was $67.8 \pm 13.0$ years and $57.2 \%$ of the study population was male. Patients were classified as $\operatorname{HFrEF}(n=1028,64 \%), \mathrm{HFmrEF}(n=305$, $19 \%)$, and HFpEF $(n=273,17 \%)$ according to LVEF. HFmrEF patients were elder than HFrEF patients but younger than HFpEF patients and the female proportion was the highest in HFpEF group followed by HFmrEF and HFrEF groups $(P<0.001$ and $P=0.03$, respectively). The prevalence of coronary artery disease was $56.7 \%$ in HFmrEF patients. It was lower than HFrEF patients $(65.2 \%)$ and higher than HFpEF patients $(41.4 \%)(P<0.001)$. The prescription of evidence-based HF drugs (Renin-Angiotensin-System blocker, beta-blocker, mineralocorticoid receptor antagonist) was similar in HFrEF and HFmrEF patients and higher than HFpEF patients. The in-hospital mortality rate was the lowest in patients with $\mathrm{HFmrEF}(1.8 \%, 7.3 \%$, and $7.5 \%$, respectively for $\mathrm{HFmrEF,} \mathrm{HFrEF}$, and $\mathrm{HFpEF}$ patients $)(P<0.001)$. Conclusion: Patients with HFmrEF has unique clinical, echocardiographic, hemodynamic, and biomarker features compared with HFrEF and HFpEF. However, patients with HFmrEF seem to be more similar to HFrEF, in terms of etiology and use of guideline recommended medical therapy.

Keywords: Acute heart failure, clinical characteristics, demographics, heart failure with mid-range ejection fraction

Received: 27-08-2019 Revised: 11-10-2019 Accepted: 02-11-2019

Published Online: 13-02-2020

\begin{tabular}{|l|l|}
\hline \multicolumn{2}{|c|}{ Access this article online } \\
\hline Quick Response Code: & Website: \\
& http:/www.ijcva.com \\
\cline { 2 - 2 } & \\
\hline
\end{tabular}

Address for correspondence: Dr. Umit Yasar Sinan Department of Cardiology, Istanbul University Cerrahpasa, Institute of Cardiology, Fatih, Istanbul, Turkey. E-mail: drumityasar@hotmail.com

This is an open access journal, and articles are distributed under the terms of the Creative Commons Attribution-NonCommercial-ShareAlike 4.0 License, which allows others to remix, tweak, and build upon the work non-commercially, as long as appropriate credit is given and the new creations are licensed under the identical terms.

For reprints contact: reprints@medknow.com

How to cite this article: Sinan UY, Gurbuz DC, Celik O, Cakmak HA, Kilic S, Inci S, et al. The clinical characteristics of acute heart failure patients with mid-range ejection fraction in Turkey: A subgroup analysis from journey HF-TR study. Int J Cardiovasc Acad 2020;6:5-11. 


\section{INTRODUCTION}

Heart failure (HF) is a clinical syndrome characterized by a wide spectrum of factors, including left ventricular (LV) structural and functional abnormalities ranging from preserved LV ejection fraction (LVEF) with normal LV size to severely reduced LVEF with marked dilatation of the left ventricle. ${ }^{[1]}$ LVEF is considered important for classifying HF patients because of differing patient demographics, prognosis, as well as response to $\mathrm{HF}$ therapies.

The 2016 European Society of Cardiology HF guideline recognized $\mathrm{HF}$ with mid-range $\mathrm{EF}$ (HFmrEF, EF $40 \%-49 \%$ ) as an entity distinct from $\mathrm{HF}$ with reduced $\mathrm{EF}(\mathrm{HFrEF}, \mathrm{EF}<40 \%)$ and preserved $\mathrm{EF}$ (HFpEF, $\mathrm{EF} \geq 50 \%$ ). ${ }^{[2]}$ In this guideline, HFmrEF was defined as HF with an EF between $40 \%$ and $49 \%$, along with symptoms and/or signs of HF, elevated levels of natriuretic peptides and evidence of other cardiac functional or structural alterations such as left atrial enlargement, LV hypertrophy or diastolic dysfunction. ${ }^{[2]}$ Clinical characteristics and clinical outcomes of HFmrEF patients are described in various cohorts..$^{[3-8]}$ Especially Organized Program to Initiate Life-saving Treatment in Hospitalized Patients with HF (OPTIMIZE-HF) and Acute Decompensated HF National Registry (ADHERE) studies lead other studies to explore the characteristics, treatment patterns, and outcomes of patients with mildly reduced LVEF. ${ }^{[9,10]}$

The percentage of HFmrEF patients is reported to be between 13\% and $24 \%{ }^{[4,7,11]}$ GWTG-HF registry has found that although the percentage of patients with $\mathrm{HFpEF}$ increased (from 33\% to 39\%) and the percentage $\mathrm{HFrEF}$ decreased (from $52 \%$ to $47 \%$ ) from 2005 to 2010, the percentage of patients with HFmrEF has remained relatively steady (between $13 \%$ and $15 \%$ ) over this period. ${ }^{[12]}$ Often considered a "gray" area or the "middle child" in HF, HFmrEF is gaining increasing attention in recent studies. ${ }^{[5]}$

In this analysis, we aimed to investigate the clinical characteristics, demographics, in-hospital management, and in-hospital outcomes of patients with HFmrEF and compare them to those with HFrEF and HFpEF in a large acute HF (AHF) cohort.

\section{Materials and Methods}

The Journey HF-TR study is a cross-sectional, multicenter, noninterventional, and observational registry ${ }^{[13]}$ The patients who were hospitalized with AHF in the intensive/coronary care units and cardiology wards of participating centers between September 2015 and September 2016 were included in our study. We enrolled a total of 1606 patients in 37 centers, in seven geographical regions of Turkey. Study centers were designed to represent the 12 territorial units of Turkey, accepted by the National Statistics Unit (NUTS 1). The inclusion criteria were being older than 18-year-old, hospitalization with AHF and accepting to give informed consent to participate in this study. The patients were diagnosed with AHF at first hospital admission by attending cardiologists and were classified according to ESC HF Guideline in 2016. Patients' demographic and clinical characteristics, clinical histories, symptoms and signs and their progress in hospital (diagnostic tests, laboratory findings, medications, length of stay, and mortality) were evaluated and recorded.

We selected patients with documented LVEF and we compared baseline demographic and clinical characteristics, clinical presentation, in-hospital intravenous and oral therapies, and in-hospital mortality among patients with HFrEF, HFmrEF, and HFpEF. HFrEF was defined as an LVEF lower than $40 \%$, typical symptoms and or signs of HF; HFmrEF was defined as an LVEF ranging between $40 \%$ and $49 \%$, typical symptoms and or signs of HF, elevated natriuretic peptides and relevant structural heart disease or diastolic dysfunction; and HFpEF was defined as an LVEF equal to or higher than $50 \%$, typical symptoms and/or signs of HF, elevated natriuretic peptides and relevant structural heart disease or diastolic dysfunction. Demographic and clinical data were recorded at or close to patient discharge, based on medical records. In-hospital mortality rates were also recorded.

Chronic kidney disease (CKD) is defined as kidney damage or glomerular filtration rate $<60 \mathrm{~mL} / \mathrm{min} / 1.73 \mathrm{~m}^{2}$ for 3 months or more, irrespective of cause. Dyslipidemia is defined as increased total or low-density lipoprotein cholesterol or being on lipid-lowering therapy. Patients whose office blood pressure (BP) is out of target range (systolic BP higher than $140 \mathrm{mmHg}$ and diastolic BP higher than $90 \mathrm{mmHg}$ ) are defined as patients with uncontrolled HT. Anemia is defined as $\mathrm{Hb}<13.0 \mathrm{~g} / \mathrm{dl}$ for males and $\mathrm{Hb}<12.0 \mathrm{~g} / \mathrm{dl}$ for females using the WHO definition. Cardiorenal syndrome is defined as the disorder of the heart and kidneys, where acute or chronic dysfunction in one organ may induce acute or chronic dysfunction of the other. We accepted infection as the cause of worsening of HF if there were signs of infection (fever, high C-reactive protein, leukocytosis, and nidus. New-onset ("de novo") HF may present acutely as a consequence of acute myocardial infarction, or in a subacute (gradual) fashion in patients with dilated cardiomyopathy who often have symptoms for weeks or months before the diagnosis becomes apparent. ${ }^{[2]}$ If patients with AHF present with elevated systolic $\mathrm{BP}(>140 \mathrm{mmHg})$, it is defined as hypertensive AHF. ${ }^{[2]}$

\section{Statistical analysis}

For baseline characteristics, categorical variables were described as numbers and percentages and continuous variables as mean \pm standard deviation, or as median with inter-quartile range if skewed. Categorical variables were compared using a Chi-square test or Fisher's exact test if any expected cell count was $<5$. One-sample Kolmogorov-Smirnov test was used to identify whether the distribution of variable was normal or not. Differences in characteristics across the three EF groups were compared with analysis of variance test for continuous variables. A value of $P<0.05$ was considered statically significant. All tests were two-sided. Analyses were performed using the Statistical Package for the Social Sciences (SPSS) software for windows, version 22.0 (IBM Corp., Armonk, NY, USA). 


\section{RESULTS}

One thousand six hundred and six patients who were diagnosed with AHF in 37 centers, in seven geographical regions of Turkey were enrolled in this study. The mean age was $67.8 \pm 13.0$ years and $57.2 \%$ of the study population was male. Most of the patients had the New York Heart Association functional capacity III or IV. The mean EF of the whole group was $32.7 \pm 14.1 \%$. Patients were classified as $\operatorname{HFrEF}(n=1028$, $64 \%)$, HFmrEF $(n=305,19 \%)$, and $\operatorname{HFpEF}(n=273,17 \%)$ according to LVEF.

The number of female and male patients was almost the same in HFmrEF patients (51.1\% and $48.9 \%$ ). The mean age of HFmrEF patients was higher than HFrEF patients but lower than HFpEF patients. The female proportion was the highest in HFpEF group followed by HFmrEF and HFrEF groups (respectively, $P<0.001$ and $P=0.03$ ). The prevalence of coronary artery disease (CAD) was $56.7 \%$ in HFmrEF patients, lower than the prevalence in HFrEF patients (65.2\%) and higher than HFpEF patients $(41.4 \%)(P<0.001)$. Atrial fibrillation $(\mathrm{AF})$ was less prevalent in HFmrEF patients $(41.8 \%)$ than the other two groups (52\% in HFrEF patients and 56.3\% in HFpEF patients, $P<0.001)$. Compared to HFrEF, not only HFmrEF patients but also HFpEF patients had higher prevalence of anemia $(P<0.001)$ and hypertension $(P<0.001)$. The other comorbidities (diabetes mellitus [DM], dyslipidemia, CKD, previous history of transient ischemic attack (TIA), or cerebrovascular attack (CVA) were similar between three groups.

Acute decompensation of chronic HF was the leading cause of hospitalization in all groups $(74.3 \%, 52.8 \%$, and $44.4 \%$ in HFrEF, HFmrEF, and HFpEF patients, respectively). De novo AHF was more common among HFmrEF patients. Fourteen percent of HFrEF patients, $23.0 \%$ of HFmrEF patients, and $20.1 \%$ of HFpEF patients were hospitalized with de novo HF diagnosis $(P=0.02)$. The main causes for hospitalization in HFmrEF patients were acute coronary syndrome (ACS) $30.5 \%$, arrhythmias $24.8 \%$, uncontrolled HT $24.2 \%$, cardio-renal syndrome $8.4 \%$, lack of compliance to therapy $8.0 \%$, and infection $4.1 \%$. HFmrEF patients had a higher prevalence of ACS as the precipitating factor compared to the other two groups $(30.5 \%$ and $19.4 \%$ for HFrEF and $12.3 \%$ for HFpEF; $P<0.01)$. Hypertensive

\begin{tabular}{|c|c|c|c|c|}
\hline Etiologies & HFrEF $(n=1028)$ & HFmrEF $(n=305)$ & HFpEF $(n=273)$ & $P$ \\
\hline De novo, $n(\%)$ & $149(14.5)$ & $70(23)$ & $55(20.1)$ & $<0.001$ \\
\hline Acute decompensated chronic HF, $n(\%)$ & $879(85.5)$ & $235(77)$ & $218(79.9)$ & $<0.001$ \\
\hline \multicolumn{5}{|l|}{ Clinical presentation } \\
\hline Decompensation of CHF, $n(\%)$ & $653(74.3)$ & $120(52.8)$ & $97(44.4)$ & $<0.001$ \\
\hline Pulmonary edema, $n(\%)$ & $85(9.7)$ & $50(22)$ & $68(31.3)$ & $<0.001$ \\
\hline Cardiogenic shock, $n(\%)$ & $40(4.6)$ & $5(2.1)$ & $5(2.2)$ & 0.07 \\
\hline HT HF, $n(\%)$ & $89(10.1)$ & $50(22)$ & $38(17.5)$ & $<0.001$ \\
\hline RV HF, $n(\%)$ & $12(1.3)$ & $3(1.1)$ & $10(4.6)$ & $<0.001$ \\
\hline \multicolumn{5}{|l|}{ Precipitants } \\
\hline ACS, $n(\%)$ & $200(19.4)$ & $30.5(93)$ & $12.3(34)$ & $<0.001$ \\
\hline Infection, $n(\%)$ & $205(20.0)$ & $13(4.1)$ & $55(20.0)$ & $<0.001$ \\
\hline Arrhythmia, $n(\%)$ & $230(22.4)$ & $75(24.8)$ & $85(31.3)$ & 0.005 \\
\hline Uncontrolled HT, $n(\%)$ & $148(14.4)$ & $74(24.2)$ & $70(25.7)$ & $<0.001$ \\
\hline Cardio-renal syndrome, $n(\%)$ & $28(2.7)$ & $26(8.4)$ & $17(6.4)$ & 0.01 \\
\hline Medical in-adherence, $n(\%)$ & $217(21.1)$ & $24(8.0)$ & $12(4.3)$ & $<0.001$ \\
\hline Age (years old) & $66.9 \pm 13.3$ & $68.6 \pm 12.6$ & $71.5 \pm 11.5$ & $<0.001$ \\
\hline Male sex, $n(\%)$ & $664(64.6)$ & 149 (48.9) & $105(38.4)$ & $<0.001$ \\
\hline $\mathrm{CAD}, n(\%)$ & $670(65.2)$ & $173(56.7)$ & $113(41.4)$ & $<0.001$ \\
\hline HT, $n(\%)$ & $639(62.2)$ & $240(78.7)$ & $200(73.1)$ & $<0.001$ \\
\hline DM Type 2, $n(\%)$ & $432(42)$ & $137(45)$ & $101(37)$ & 0.148 \\
\hline Smoking, $n(\%)$ & $293(28.5)$ & $83(27.3)$ & $43(15.7)$ & $<0.001$ \\
\hline $\mathrm{AF}, n(\%)$ & $535(52)$ & $127(41.8)$ & $154(56.3)$ & $<0.001$ \\
\hline $\mathrm{HL}, n(\%)$ & $292(28.4)$ & $93(30.5)$ & $72(26.5)$ & 0.580 \\
\hline TIA/CVA, $n(\%)$ & $123(12)$ & $27(8.9)$ & $22(8.2)$ & 0.197 \\
\hline $\mathrm{CKD}, n(\%)$ & $306(29.8)$ & $78(25.5)$ & $68(25)$ & 0.167 \\
\hline Anemia, $n(\%)$ & $421(41)$ & $189(62)$ & $172(63)$ & $<0.001$ \\
\hline $\mathrm{PAD}, n(\%)$ & $81(7.9)$ & $119(3.9)$ & $10(3.7)$ & 0.02 \\
\hline
\end{tabular}

ACS: Acute coronary syndrome, AF: Atrial fibrillation, CAD: Coronary artery disease, CHF: Chronic heart failure, CKD: Chronic kidney disease, CVA: Cerebrovascular accident, DM: Diabetes mellitus, HF: Heart failure, HFmrEF: Heart failure mid-range ejection fraction, HFpEF: Heart failure with preserved ejection fraction, HFrEF: Heart failure with reduced ejection fraction, HL: Hyperlipidemia, HT: Hypertension, LVEF: Left ventricular ejection fraction, PAD: Peripheral artery disease, RV: Right ventricle, TIA: Transient ischemic attack 
HF was also more common in HFmrEF patients $(22 \%)$. While uncontrolled hypertension and cardio-renal syndrome were much more seen in HFmrEF $(24.2 \%$ and $8.2 \%)$ and HFpEF (25.7\% and 6.4\%) patients compared to HFrEF (14.4\% and $2.7 \%)$ patients $(P<0.01$ and $P=0.01)$, lack of compliance to therapy was seen much more in HFrEF patients $(21.1 \%$ and 8.0 for HFmrEF and $4.3 \%$ for HFpEF; $P<0.01)$. Infection was the least precipitating factor for hospitalization in $\mathrm{HFmrEF}$ patients $(4.1 \%$; $P<0.01)$ [Table 1].

Vital signs of the three groups are compared in Table 2. The mean N-terminal pro-brain natriuretic peptide level was 8902 pg/ml (7923-9932) in HFrEF patients, 6030 pg/ml (4317-7742) in HFmrEF, and $4406 \mathrm{pg} / \mathrm{ml}$ (3334-5479) in HFpEF patients $(P<0.001)$ [Table 2]. At discharge, the natriuretic peptide levels of the three groups were similar.
Regarding medical management, diuretic therapy was more frequently used in HFrEF and HFmrEF patients than in HFpEF patients at admission [Table 3]. Compared to HFmrEF and HFpEF patients, HFrEF patients were characterized by higher use of mineralocorticoid-receptor antagonists (MRAs) $(P=0.001)$. There was a significantly larger number of patients with a history of cardiac device implantation (implantable cardioverter-defibrillator or cardiac resynchronization therapy) in HFrEF group. At hospital discharge, guideline-recommended medical therapy prescription was done more efficiently than first admission in all groups [Table 3]. The prescription of evidence-based HF drugs (RAAS blocker, beta-blocker, MRA) was similar in HFrEF and HFmrEF groups, which was higher than HFpEF group.

In-hospital mortality rate was least in HFmrEF patients $(1.8 \%, 7.3 \%$, and $7.5 \%$, respectively, for $\mathrm{HFmrEF}, \mathrm{HFrEF}$, and HFpEF patients) $(P<0.001)$.

\section{Table 2: Physical examination findings, laboratories, and QRS duration of heart failure subgroups}

\begin{tabular}{|c|c|c|c|c|}
\hline Physical examination & HFrEF & HFmrEF & HFpEF & $P$ \\
\hline SBP at admission $(\mathrm{mmHg})$ & $123 \pm 28$ & $135 \pm 32$ & $139 \pm 39$ & $<0.001$ \\
\hline $\mathrm{HR}$ at admission (bpm) & $93 \pm 23$ & $93 \pm 24$ & $98 \pm 26$ & 0.04 \\
\hline QRS duration (msn) & $110 \pm 41$ & $99 \pm 28$ & $100 \pm 19$ & $<0.001$ \\
\hline $\mathrm{EF}(\%)$ & $27.1 \pm 7$ & $42.5 \pm 4.2$ & $55.2 \pm 4.8$ & $<0.001$ \\
\hline GFR at admission (ml/min) & $50.4 \pm 29$ & $47.6 \pm 33.5$ & $49.6 \pm 30.5$ & 0.398 \\
\hline NT-proBNP at admission $(\mathrm{pg} / \mathrm{ml})$ & $8902(7923-9932)$ & $6030(4317-7742)$ & 4406 (3334-5479) & $<0.001$ \\
\hline HR at discharge (bpm) & $73.1 \pm 20.7$ & $64.6 \pm 30$ & $72.8 \pm 24.5$ & $<0.001$ \\
\hline SBP at discharge $(\mathrm{mmHg})$ & $107 \pm 31.1$ & $97.7 \pm 47.5$ & $110.8 \pm 33.3$ & $<0.001$ \\
\hline NT-proBNP at discharge $(\mathrm{pg} / \mathrm{ml})$ & $3817(2875-4759)$ & $2306(1527-3086)$ & $2028(605-3451)$ & 0.248 \\
\hline
\end{tabular}

EF: Ejection fraction, GFR: Glomerular filtration rate, HF: Heart failure, HFmrEF: Heart failure mid-range ejection fraction, HFpEF: Heart failure with preserved ejection fraction, HFrEF: Heart failure with reduced ejection fraction, HR: Heart rate, NT-proBNP: N-terminal pro B-type natriuretic peptide, SBP: Systolic blood pressure

\begin{tabular}{|c|c|c|c|c|}
\hline & HFrEF $(n=1028)$ & HFmrEF $(n=305)$ & HFpEF $(n=273)$ & $P$ \\
\hline \multicolumn{5}{|l|}{ Medication at admission } \\
\hline ACEIs/ARBs, $n(\%)$ & $672(65.4)$ & $192(63.1)$ & $127(46.4)$ & $<0.001$ \\
\hline $\mathrm{BB}, n(\%)$ & $781(76)$ & $203(66.6)$ & $153(55.9)$ & $<0.001$ \\
\hline Diuretics, $n(\%)$ & $763(74.2)$ & $207(68)$ & $168(61.6)$ & $<0.001$ \\
\hline MRAs, $n(\%)$ & $441(42.9)$ & $97(31.9)$ & $81(29.8)$ & 0.001 \\
\hline CCBs, $n(\%)$ & $43(4.2)$ & $30(9.9)$ & $40(14.5)$ & $<0.001$ \\
\hline Digoksin, $n(\%)$ & $220(21.4)$ & $68(22.3)$ & $45(16.4)$ & 0.238 \\
\hline CRT/ICD, $n(\%)$ & $221(21.5)$ & $24(7.8)$ & $14(4.4)$ & $<0.001$ \\
\hline \multicolumn{5}{|l|}{ Medication at discharge } \\
\hline ACEIs/ARBs, $n(\%)$ & $828(80.5)$ & $269(88.3)$ & $167(61.2)$ & $<0.001$ \\
\hline $\mathrm{BB}, n(\%)$ & $923(89.8)$ & $267(87.6)$ & $203(74.3)$ & $<0.001$ \\
\hline Diuretics, $n(\%)$ & $874(85)$ & $216(70.9)$ & $222(81.3)$ & $<0.001$ \\
\hline MRA, $n(\%)$ & $642(62.5)$ & $186(61)$ & $98(35.8)$ & $<0.001$ \\
\hline $\mathrm{CCB}, n(\%)$ & $43(4.2)$ & $30(9.9)$ & $40(14.5)$ & $<0.001$ \\
\hline Digoksin, $n(\%)$ & $240(23.3)$ & $64(20.9)$ & $67(24.6)$ & 0.07 \\
\hline LOS (days) & 11.7 & 22.5 & 10 & $<0.001$ \\
\hline Mortality, $n(\%)$ & $75(7.3)$ & $5(1.8)$ & $20(7.5)$ & 0.01 \\
\hline
\end{tabular}

ACEIs: Angiotensin converting enzyme inhibitors, ARBs: Angiotensin receptor blockers, BB: Beta blocker, CCB: Calcium channel blockers, CRT: Cardiac resynchronization therapy, HF: Heart failure, LOS: Length of hospital stay, Heart failure mid-range ejection fraction, HFpEF: Heart failure with preserved ejection fraction, HFrEF: Heart failure with reduced ejection fraction, ICD: Implantable cardiovertor defibrillator MRAs: Mineralocorticoid receptor blockers 


\section{Discussion}

In this subgroup analysis of the Journey HF-TR registry, it has been shown that nearly one-fifth of patients $(19 \%)$ who were hospitalized with a diagnosis of AHF had HFmrEF. In GTWG-HF registry, which was performed in $>40,000$ Medicare patients, $14 \%$ of patients fell into HFmrEF category. ${ }^{[12]}$ The percentage of HFmrEF patients ranges between $13 \%$ and $24 \%$ in various cohorts. ${ }^{[4,7,11]}$ In our study, $36 \%$ of patients had either mid-range or preserved LVEF. This prevalence is lower than the two largest AHF registries from US, ADHERE, OPTIMIZE-HF, and European Euro-HF Survey I, in which the prevalence of LVEF $\geq 40 \%$ ranged from $40 \%$ to $55 \% .{ }^{[9,14,15]}$ However, the prevalence was higher than Turkish HF registry (SELFIE-TR) ${ }^{[16]}$ The total number of patients HFrEF, HFmrEF, and HFpEF was 801 (75\%), $176(16.7 \%)$, and $77(7.3 \%)$, respectively, in SELFIE-TR. ${ }^{[16]}$

The prevalence of HF in Turkey was reported as $6.9 \%$ in the HAPPY trial ${ }^{[17]}$ and the population older than 35 years in Turkey was reported as 29.6 million according to 2010 records ${ }^{[18]}$ The percentage of the patients with HFmrEF is $19 \%$, suggesting that approximately 460,560 individuals in Turkey have HFmrEF.

Given the differences in demography, clinical presentation, etiology, and prognosis in the three groups, some authors suggest that HFmrEF has a phenotype closer to HFpEF, whereas others consider it to be closer to HFrEF. An analysis of 41,267 patients in OPTIMIZE-HF analyzed patients hospitalized with HF according to LVEF group showed that patients with LVEF between $40 \%$ and $50 \%$ were more similar to patients with HFpEF. ${ }^{[9]}$ Moreover, in GTWG-HF registry, patients with HFmrEF had clinical characteristics (older age, female sex, comorbidities as HT, chronic obstructive pulmonary disease (COPD), DM, laboratory values as creatinine, BNP, troponin and medication use as beta-blockers, RAAS blockers) more similar to those of HFpEF cohorts. ${ }^{[12]}$ On the other hand, CAD was the morbidity which was more similar between HFmrEF and HFrEF patients. ${ }^{[12]}$ In conclusion, HFmrEF may resemble HFpEF with an exceptional etiology of ischemia, where it resembles HFrEF more. ${ }^{[12]}$ Contrarily, in a paper by Nauta et al. evaluating what we learned about HFmrEF 1 year after its introduction, it was concluded that although HFmrEF patients are considered to be the "middle child," it seems to be more similar to HFrEF, in terms of ischemic etiology, biomarker profile, and response to treatment. ${ }^{[19]}$

In our study, patients with HFmrEF were younger and more predominantly male compared to those with HFpEF. This was compatible with other studies. ${ }^{[5,11,20-23]}$ Several CV risk factors (DM, CKD, dyslipidemia, TIA, CVA) were shared among HFmrEF, HFrEF, and HFpEF, but patients with HFmrEF were more likely to have HT (78\%) and de novo AHF (23\%) compared to those with HFrEF and HFpEF. The HT prevalence in patients with HFmrEF was higher than other registries (between $60 \%$ and $77 \%$ ). ${ }^{[5]}$ Of note, patients with HFmrEF were more likely to have ischemic heart disease (57\%) compared with those with HFpEF (41\%) The ratio of patients who had ischemic heart disease in HFmrEF and HFrEF (65\%) groups were similar. In the Sweedish HF registry, the percentages of IHD were $60 \%$ for HFrEF, $61 \%$ for HFmrEF, and $52 \%$ for HFpEF. ${ }^{[24]}$ In ESC HF Long-Term Registry, the etiology was ischemia for $48.6 \%$ of HFrEF patients, $41.8 \%$ for HFmrEF patients, but only in $23.7 \%$ for HFpEF patients. ${ }^{[25]}$ An extensive post hoc analysis of the TIME-CHF trial showed ischemic etiology was $58.2 \%, 56.5 \%, 31.3 \%$ for HFrEF, HFmrEF, and HFpEF, respectively. ${ }^{[21]}$ Therefore, in terms of etiology HFmrEF is more like HFrEF than HFpEF. The AF prevalence is lower in patients with $\mathrm{HFmrEF}(42 \%)$ compared to those with HFrEF (52\%) and HFpEF (56\%). The prevalence of $\mathrm{AF}$ in Sweedish HF registry was $65 \%, 60 \%$, and $53 \%$ in HFpEF, HFmrEF, and HFrEF, respectively. ${ }^{[24]}$ In accordance with our study, Löfman et al. found that CKD was associated with similar covariates regardless of EF along the EF spectrum in the Swedish HF registry. ${ }^{[22]}$ Chioncel et al. ${ }^{[25]}$ showed that COPD, liver disease, and CKD were more common in HFrEF, but in Rickenbacher's study three EF strata had a comparably high burden of comorbidities. ${ }^{[21]}$

In patients with HFmrEF, ACS, arrhythmias, and uncontrolled HT were more often precipitating factors for HF hospitalization compared with other HF groups. Infection was the least precipitating factor in these patients. Systolic BP and natriuretic peptide levels of patients with HFmrEF also fell in between those of HFrEF and HFpEF.

The ESC HF Long-Term Registry gives precise information in the current practice regarding HF medication. The use of beta-blockers and ACE inhibitors was around $90 \%$ in both HFrEF and HFmrEF, compared to approximately $75 \%$ in patients with HFpEF. ${ }^{[25]}$ Percentages in Swedish HF registry were comparable. ${ }^{[2]}$ Use of MRAs was higher in the ESC HF Long-Term Registry: 70\% in HFrEF, 55\% in HFmrEF, and $35 \%$ in HFpEF compared to Swedish HF Registry. ${ }^{[24,25]}$ In our study, use of RAAS blockers, beta-blockers, and MRAs was similar in those with HFrEF and HFmrEF, higher than the use of these medications in patients with HFpEF. At discharge, guideline-recommended medical therapy prescription was higher than first admission in all groups. Interestingly, prescription of diuretic was the lowest in patients with HFmrEF at discharge. As you can see in Table 1, the AF prevalence is the highest in HFpEF patients and it is followed by HFrEF and HFmrEF patients. Hence, digoksin may be used higher than expected in HFpEF and HFmrEF patients due to heart rate-limiting effect.

Among patients in ADHERE, in-hospital mortality was 4.7\% in patients with LVEF $<25 \%, 3.4 \%$ in patients with LVEF between $25 \%$ and $40 \%, 3.2 \%$ in those with LVEF between $41 \%$ and $54 \%$ and $3.0 \%$ in those with LVEF $\geq 55 \%{ }^{\left[{ }^{[10]}\right.}$ Kapoor et al. found in hospital mortality of $3.2 \%$ for $\mathrm{HFrEF}, 2.6 \%$ for HFmrEF, and $3.0 \%$ for HFpEF in the GTWG-HF Registry, which included 98,825 adult patients hospitalized for new or worsening HF. ${ }^{[23]}$ The in-hospital mortality rate of those with 
HFrEF (7.3\%) and HFpEF (7.5\%) was higher in our study than ADHERE and GTWG-HF registry. However, patients with HFmrEF in our study had lower in hospital mortality rate $(1.8 \%)$ than these two registry cohorts.

Although there are numerous studies that investigated the clinical characteristics, demographics, compliance with guideline-recommended medical therapy, and the prognosis of stable HFmrEF patients, only a few studies considered the features of HFmrEF patients in AHF population. . $^{[3,4,7,11,23,26,27]}$ The study is unique in the sense that it investigates the characteristics of HFmrEF in the setting of AHF compared to HFrEF and HFmrEF in Turkey.

\section{Study limitation}

The registry data were based on documentation of medical history only. Information about management during hospitalization and follow-up was not obtained. Therefore, readmission rate of patients after discharge is unknown. Laboratory parameters, biomarkers, dosage, and the duration of HF medications should be standardized. In addition, medication dosage was not recorded, and hence, we were not sure if the patients were taking appropriate doses of HF medications. The last but not the least limitation of our study was the lack of natriuretic peptide levels in $60 \%$ of the study population due to limited local resources.

\section{ConcLusion}

After the introduction of HFmrEF as a separate category, several registries were conducted to define these patients' characteristics, demographics, and prognosis. Several interesting insights have been yielded. In conclusion, patients with HFmrEF have unique clinical, echocardiographic, hemodynamic, and biomarker features compared to patients with HFrEF and HFpEF. However, patients with HFmrEF seem to be more similar to HFrEF, in terms of (ischemic) etiology and the use of guideline-recommended medical therapy.

\section{Financial support and sponsorship}

Nil.

\section{Conflicts of interest}

There are no conflicts of interest.

\section{References}

1. Yancy CW, Jessup M, Bozkurt B, Butler J, Casey DE Jr., Drazner MH, et al. $2013 \mathrm{ACCF} / \mathrm{AHA}$ guideline for the management of heart failure: Executive summary: A report of the American College of Cardiology Foundation/American Heart Association task force on practice guidelines. Circulation 2013;128:1810-52.

2. Ponikowski P, Voors AA, Anker SD, Bueno H, Cleland JG, Coats AJ, et al. 2016 ESC guidelines for the diagnosis and treatment of acute and chronic heart failure: The task force for the diagnosis and treatment of acute and chronic heart failure of the European Society of Cardiology (ESC). Developed with the special contribution of the Heart Failure Association (HFA) of the ESC. Eur J Heart Fail 2016;18:891-975.

3. Cheng RK, Cox M, Neely ML, Heidenreich PA, Bhatt DL, Eapen ZJ, et al. Outcomes in patients with heart failure with preserved, borderline, and reduced ejection fraction in the medicare population. Am Heart J 2014;168:721-30.

4. Coles AH, Tisminetzky M, Yarzebski J, Lessard D, Gore JM, Darling CE, et al. Magnitude of and prognostic factors associated with 1-year mortality after hospital discharge for acute decompensated heart failure based on ejection fraction findings. J Am Heart Assoc 2015;4. pii: e002303.

5. Lam CS, Solomon SD. The middle child in heart failure: Heart failure with mid-range ejection fraction (40-50\%). Eur J Heart Fail 2014;16:1049-55.

6. Lam CS, Teng TH. Understanding heart failure with mid-range ejection fraction. JACC Heart Fail 2016;4:473-6.

7. Kapoor JR, Kapoor R, Ju C, Heidenreich PA, Eapen ZJ, Hernandez AF, et al. Precipitating clinical factors, heart Failure Characterization, and outcomes in patients hospitalized with heart failure with reduced, borderline, and preserved ejection fraction. JACC Heart Fail 2016;4:464-72.

8. Coles AH, Fisher K, Darling C, Yarzebski J, McManus DD, Gore JM, et al. Long-term survival for patients with acute decompensated heart failure according to ejection fraction findings. Am J Cardiol 2014; $114: 862-8$.

9. Fonarow GC, Stough WG, Abraham WT, Albert NM, Gheorghiade M, Greenberg BH, et al. Characteristics, treatments, and outcomes of patients with preserved systolic function hospitalized for heart failure: A report from the OPTIMIZE-HF registry. J Am Coll Cardiol 2007;50:768-77.

10. Galvao M, Kalman J, DeMarco T, Fonarow GC, Galvin C, Ghali JK, et al. Gender differences in in-hospital management and outcomes in patients with decompensated heart failure: Analysis from the acute decompensated heart failure national registry (ADHERE). J Card Fail 2006;12:100-7.

11. Tsuji K, Sakata Y, Nochioka K, Miura M, Yamauchi T, Onose T, et al. Characterization of heart failure patients with mid-range left ventricular ejection fraction-a report from the CHART-2 study. Eur J Heart Fail 2017;19:1258-69.

12. Steinberg BA, Zhao X, Heidenreich PA, Peterson ED, Bhatt DL, Cannon CP, et al. Trends in patients hospitalized with heart failure and preserved left ventricular ejection fraction: Prevalence, therapies, and outcomes. Circulation 2012;126:65-75.

13. Sinan ÜY, Ekmekçi A, Özbay B, Akyıldız Akçay F, Bekar L, Koza Y, et al. The real-life data of hospitalized patients with heart failure: On behalf of the journey HF-TR study investigators. Anatol J Cardiol 2019;21:25-30.

14. Yancy CW, Lopatin M, Stevenson LW, De Marco T, Fonarow GC; ADHERE Scientific Advisory Committee and Investigators. et al. Clinical presentation, management, and in-hospital outcomes of patients admitted with acute decompensated heart failure with preserved systolic function: A report from the acute decompensated heart failure national registry (ADHERE) database. J Am Coll Cardiol 2006;47:76-84.

15. Cleland JG, Swedberg K, Follath F, Komajda M, Cohen-Solal A, Aguilar JC, et al. The Euroheart failure survey programme - A survey on the quality of care among patients with heart failure in Europe. Part 1: Patient characteristics and diagnosis. Eur Heart J 2003;24:442-63.

16. Yılmaz MB, Çelik A, Çavuşoğlu Y, Bekar L, Onrat E, Eren M, et al. Snapshot evaluation of heart failure in turkey: Baseline characteristics of SELFIE-TR. Turk Kardiyol Dern Ars 2019;47:198-206.

17. Değertekin M, Erol C, Ergene O, Tokgözoğlu L, Aksoy M, Erol MK, et al. Heart failure prevalence and predictors in turkey: HAPPY study. Turk Kardiyol Dern Ars 2012;40:298-308.

18. Available from: http://www.tuik.gov.tr. [Last accessed on 2019 May].

19. Nauta JF, Hummel YM, van Melle JP, van der Meer P, Lam CS, Ponikowski $\mathrm{P}$, et al. What have we learned about heart failure with mid-range ejection fraction one year after its introduction? Eur J Heart Fail 2017;19:1569-73.

20. Farmakis D, Simitsis P, Bistola V, Triposkiadis F, Ikonomidis I, Katsanos S, et al. Acute heart failure with mid-range left ventricular ejection fraction: Clinical profile, in-hospital management, and short-term outcome. Clin Res Cardiol 2017;106:359-68.

21. Rickenbacher P, Kaufmann BA, Maeder MT, Bernheim A, Goetschalckx K, Pfister O, et al. Heart failure with mid-range ejection 
fraction: A distinct clinical entity? Insights from the trial of intensified versus standard medical therapy in elderly patients with congestive heart failure (TIME-CHF). Eur J Heart Fail 2017;19:1586-96.

22. Löfman I, Szummer K, Dahlström U, Jernberg T, Lund LH. Associations with and prognostic impact of chronic kidney disease in heart failure with preserved, mid-range, and reduced ejection fraction. Eur J Heart Fail 2017;19:1606-14.

23. Kapoor JR, Kapoor R, Ju C, Heidenreich PA, Eapen ZJ, Hernandez AF, et al. Precipitating clinical factors, heart Failure Characterization, and outcomes in patients hospitalized with heart failure with reduced, borderline, and preserved ejection fraction. JACC Heart Fail 2016;4:464-72.

24. Koh AS, Tay WT, Teng TH, Vedin O, Benson L, Dahlstrom U, et al. A comprehensive population-based characterization of heart failure with mid-range ejection fraction. Eur J Heart Fail 2017;19:1624-34.

25. Chioncel O, Lainscak M, Seferovic PM, Anker SD, Crespo-Leiro MG, Harjola VP, et al. Epidemiology and one-year outcomes in patients with chronic heart failure and preserved, mid-range and reduced ejection fraction: An analysis of the ESC heart failure long-term registry. Eur J Heart Fail 2017;19:1574-85.

26. Rastogi A, Novak E, Platts AE, Mann DL. Epidemiology, pathophysiology and clinical outcomes for heart failure patients with a mid-range ejection fraction. Eur J Heart Fail 2017;19:1597-605.

27. Özlek B, Özlek E, Ağuş HZ, Tekinalp M, Kahraman S, Çil C, et al. Patients with HFpEF and HFmrEF have different clinical characteristics in Turkey: A multicenter observational study. Eur J Intern Med 2019;61:88-95. 